\title{
A NEW INTERFERENTIAL DILATOMETER
}

\author{
By Irwin G. Priest
}

CONTENTS

I. Introduction . . . Page

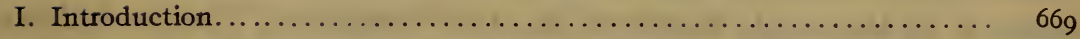

II. Description of instrument................................. $6_{70}$

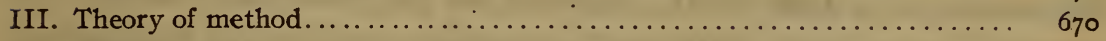

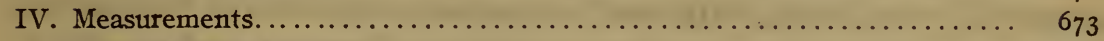

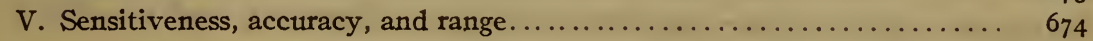

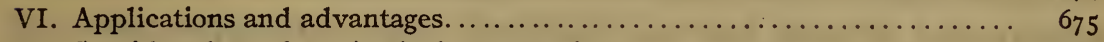

VII. Considerations of mechanical construction................... 677

\section{INTRODUCTION}

Interferential measurements of displacement or change in length by use of the interference fringes due to two nearly parallel plane mirrors have heretofore been made almost, if not quite, exclusively by means of observations on the displacement of the fringes. ${ }^{1}$ In r9r 2, the author designed a dilatometer to measure small changes in length by the change in width of interference fringes and thereby obtained a number of advantages in special cases. An instrument according to this design was later constructed in the Bureau of Standards optical shop. ${ }^{2}$ Although a few preliminary tests of the use of this method in determining differential thermal expansions were made with improvised apparatus in 1912, and reported to the Bureau of Standards scientific staff, ${ }^{3}$ no practical use was made of it for several years; and it was not thought worth while to publish anything concerning the new instrument or method until they had been thoroughly tried in practice. Such practical tests have now been made and, indeed, the instrument and method are now used regularly at the Bureau of Standards, ${ }^{4}$ in tests of thermal expansion. Some account of its use in this field has been given to the Philosophical Society of Washington, and the American Physical Society, ${ }^{5}$ but no adequate description of the instrument has yet been published.

\footnotetext{
1 Fizeau, Ann. de Chim. et de Phy. (4), 2, pp. 146-15x; 1864. Pulfrich, Zeit. für Inst., 13, p. $365 ; 1893$.

By John Clacey.

Irwin G. Priest and I. B. Olmstead, Bur. Standards Scientific Staff Meeting, Nov. r, rgr2. (Not published.)

1 By C. G. Peters. See, for example, Souder and Peters, An Investigation of the Physical Properties of Dental Materials, forthcoming B. S. Tech. Paper.

- Priest and Peters, Jour. Wash. Acad. of Sci., Aug. 19, 1917, p. 475. Peters and Priest, and Peters and Souder, Am. Phy. Soc. New York Meeting, Mar. I, I9I9. $143444^{\circ}-20$ 
A general familiarity with the literature of interferometry indicates that methods such as described in this paper have been used very little if at all, and it seems probable that the instrument to be described is the first of its kind to be constructed. While making the final revision of this paper, the author has noticed for the first time a suggestion by Pulfrich ${ }^{6}$ that the number of fringes. between two fixed lines on one mirror affords a very accurate measure of the angle between the two mirrors. This is, indeed, the essential feature of the method to be described, but it has apparently not met with much practical application heretofore. An examination of the subject in Science Abstracts and the Fortschritte der Physik has not disclosed any such applications of Pulfrich's important and valuable suggestion.

On account of the uniqueness of the design and the fact that the instrument now gives promise of being useful in attacking various problems in thermal expansion, elasticity, change in length due to magnetization, "critical points," shrinkage or expansion due to change in moisture content, etc., it now seems desirable to publish a definite description of this dilatometer and the theory of its use.

\section{DESCRIPTION OF INSTRUMENT}

The instrument may, of course, assume somewhat varied mechanical forms. That perhaps best adapted to explanation is shown in Fig. I, which is nearly the same, except for its exact dimensions and the shape of the sample, as the model actually constructed by Mr. Clacey, and now in practical use at the Bureau.

\section{THEORY OF METHOD}

Referring to Fig. I, the theory of the method may be explained as follows:

Let the apparatus be illuminated by monochromatic light from above. An observer looking down in the direction $O O$ will see a system of interference fringes as shown in the Plan, Fig. $I$, appearing to lie in the plane $b b$ of the interferometer mirror; that is, the fringes and the reference lines on the mirror can be simultaneously focused by a suitable optical instrument. These fringes are due to the interference of the light reflected from the surfaces $c c$ and $b b$, which inclose a wedge-shaped space regarded as "thick" (O.I to $0.3 \mathrm{~mm}$ ) compared to the wave length. They thus belong to the category of so-called "Fringes of thick 
plates." "Such fringes were first described by Fizeau and are known as "Fizeau's curves of equal thickness."." They are the same as used in the Fizeau-Abbe dilatometer."

Numerous elaborate treatments of such interference phenomena have been published ${ }^{10}$, but the reader not already familiar with them and desiring a brief elementary description and explanation is referred to textbooks by Lummer and Edser. ${ }^{11}$

If both the cover plate and the base plate are of glass or quartz they should not be silvered. If the base plate is of polished metal, the surface $c c$ should be coated with a thin semitransparent film of some metal to improve the visibility of the fringes.

In Fig. $\mathrm{x}, a a$ is the trace of a plane through the point of observation and perpendicular to the intersection of the mirror planes $c c$ and $b b$ produced; that is, also perpendicular to $S S$. The fringes which are nearly straight and indeed sometimes called "straight" fringes are, accurately speaking, curves convex toward the intersection of $c c$ and $b b$ and normal to $a a$.

If $L_{x}$, the length of the sample, is slightly greater (a few microns) than $L_{\mathbf{s}}$, the perpendicular from $S$ to the plane $b^{\prime} b^{\prime}$ parallel to plane $b b$, the fringes will have the curvature qualitatively indicated in the figure. If $L_{\mathrm{x}}$ is slightly less than $L_{\mathrm{s}}$ the curvature will be reversed. If $L_{\mathrm{x}}$ is exactly equal to $L_{\mathrm{s}}$ the fringes become perfect circles ("Haidinger rings") ${ }^{12}$ apparently at an infinite distance from the observer, in which case no measurements can be made by the method proposed. Measurements are made for the condition of a difference of a few microns in the lengths $L_{x}$ and $L_{s}$. In this case small segments of the fringes near the axis $a a$ are apparently straight and parallel to $s s$ and $x x$. Let $L_{x}$ be adjusted so that a few straight fringes (say 5 to 20) appear between the lines ss and $x x$. If, for any cause, the difference in the lengths $L_{\mathrm{s}}$ and $L_{\mathrm{x}}$ then changes, the number of fringes between $s s$ and $x x$ will change; and the change in $L_{3}-L_{x}$ can be computed from this change in the number of fringes.

7 Edser, Light for Students, p. 4r4.

${ }^{8}$ Müller-Pouillet, Lehrbuch der Physik, roth ed., 2, book 3 (Optik), p. 747 .

- Fizeau, Ann. de Chim. et de Phy. (4), 2, pp. I46-151; 1864. Pulfrich, Zeit. für Instk., 13, p. $365 ; 1893$.

${ }^{10}$ Michelson, Phil. MIag. (5), 13, p. 236; r882. Feussner, Winklemann's Handbuch der Physik. Pockington, Camb. Phil. Soc. Proc., 11, p. ro5; 1901. Drew, Phy. Rev., 15, p. 226; 1902. Bartsch, Diss. Marburg; 1910. Wetthauer, Diss. Marburg; 19Ir. Gehrcke and Janicki, Ann. der Phy. (4), 39, p. 43 I; 19 r2.

Pahlen, Ann. der Phy. (4), 39, p. 1567; r9r2. Janicki, Ann. der Phy. (4), 40, p. 493; r913.

11 Lummer, Müller-Pouillet Lehrbuch, loc. cit. Edser, Light for Students, pp. 416-418; 1907.

12 Haidinger, Pogg. Ann., 96, pp. 453-468; 1855. Also Rayleigh, Phil. Mag., 12, p. 489; 1906. 
The equation for this computation may be given in the following terms:

$\lambda \equiv$ wave length of light used.

$D \equiv$ perpendicular distance from $X$, bearing point of sample on plane $c c$, to knife-edge $S S$.

$d \equiv$ perpendicular distance between lines $s s$ and $x x$.

$n_{x_{1}} \equiv$ order of interference at $x x$ before the change in $L_{\mathrm{s}}-L_{\mathrm{x}}$ to be measured.

$n_{\mathrm{B} 1} \equiv$ order of interference at ss before the change in $L_{\mathrm{s}}-L_{\mathrm{x}}$ to be measured.

$n_{x_{2}}$ and $n_{\mathrm{s}_{2}} \equiv$ analogous meanings after the change.

$\delta n_{1} \equiv n_{\mathrm{x}_{1}}-n_{\mathrm{s} 1}$.

$\delta n_{2} \equiv n_{x 2}-n_{\mathrm{s} 2}$.

$L_{\mathrm{s}} \equiv$ perpendicular distance from $S$ to plane $b^{\prime} b^{\prime}$ through base of sample and parallel to $b b$.

$L_{\mathbf{x}} \equiv$ length of sample (perpendicular distance from $X$ to plane $\left.b^{\prime} b^{\prime}\right)$.

$L_{\mathrm{s} 1} \equiv L_{\mathrm{s}}$ before the change to be measured. $L_{\mathrm{s} 2} \equiv L_{\mathrm{s}}$ after the change.

$L_{x_{1}} \equiv L_{x}$ before the change to be measured. $L_{x_{2}} \equiv L_{x}$ after the change.

$\Delta L_{\mathrm{s}} \equiv L_{\mathrm{s} 2}-L_{\mathrm{s} 1}$.

$\Delta L_{\mathrm{x}} \equiv L_{\mathrm{x}_{2}}-L_{x_{1}}$.

Then, in general,

$$
\Delta L_{\mathrm{x}}=\Delta L_{\mathrm{s}}+\frac{\lambda \cdot D}{2 d}\left(\delta n_{2}-\delta n_{1}\right) .
$$

The derivation of the foregoing equation ( $I$ ) is very simple, as may be shown with the aid of Fig. IA, which shows in a very exaggerated way the angle between the mirrors $c c$ and $b b$ which are nearly but not quite parallel. Let $t_{\mathrm{s}}$, perpendicular to $b b$, be the distance between $c c$ and $b b$ at reference line $s$. Let $t_{x}$, perpendicular to $b b$, be the distance between $c c$ and $b b$ at reference line $x$. From the proportion of sides in similar triangles,

$$
\frac{L_{\mathrm{x}}-L_{\mathrm{s}}}{t_{\mathrm{x}}-t_{\mathrm{s}}}=\frac{D}{d}
$$

It is also obvious that

$$
t_{\mathrm{x}}-t_{\mathrm{s}}=\frac{\lambda \cdot \delta n}{2}
$$

Substituting (3) in (2),

$$
L_{\mathbf{x}}-L_{\mathrm{s}}=\frac{\lambda \cdot D \cdot \delta n}{2 d}
$$


Or, in the two particular cases,

$$
L_{\mathrm{x}_{1}}=L_{\mathrm{B} 1}+\frac{\lambda \cdot D \cdot \delta n_{1}}{2 d}
$$

and

$$
L_{x_{2}}=L_{\mathrm{B} 2}+\frac{\lambda \cdot D \cdot \delta n_{2}}{2 d}
$$

From (5) and (6) and the definitions of $\Delta L_{x}$ and $\Delta L_{s}$ above,

$$
\Delta L_{\mathrm{x}}=\Delta L_{\mathrm{s}}+\frac{\lambda \cdot D}{2 d}\left(\delta n_{2}-\delta n_{1}\right)
$$

which is the equation to be derived.

\section{MEASUREMENTS}

$\lambda$ is a known constant. $D, d, \delta n_{1}$ and $\delta n_{2}$ must be measured.

$d$ is readily determined by a traveling microscope or Fraunhofer micrometer.

$D$ is determined as follows: Lightly silver the surface $c c$. Place it on its bearings, $S$ and $X$ (Fig. I), and slide it a few millimeters in a direction accurately parallel to $S S$, the sample being, meanwhile, held firmly in contact with the base plate in its V-notch as shown in the figure. The bearing points will leave fine traces in the silver film. The distance between these traces $(=D)$ may then be determined in the same way as $d$.

If samples are made of a constant standard diameter, the distance $D$ becomes an instrument constant for all tests; and, indeed, $\frac{\lambda \cdot D}{2 d}$ becomes an instrument constant, say $K$, to be computed once for all, and we have

$$
\Delta L_{\mathrm{x}}=\Delta L_{\mathrm{s}}+K\left(\delta n_{2}-\delta n_{1}\right)
$$

If it is desired to work with samples of various diameters, a graph or table of $K$ as a function of the sample diameter will facilitate the computations.

It is, of course, not absolutely necessary that the sample be a cylinder. This has been chosen as, in many cases, the simplest form to prepare and as well adapted to facilitate the automatic adjustment of $D$. In special cases where some other form of sample must perforce be used, other methods of determining $D$ will readily occur to users of the instrument.

$\delta n_{1}$ and $\delta n_{2}$ are obtained as the direct result of observation by counting the whole number of fringes in each case between ss and 
$x x$, adding to this counted number the fractions of a fringe-width at $s s$ and $x x$, and prefixing algebraic signs according to the following rule:

+ , if the center of curvature lies on the side $x x$.

- , if the center of curvature lies on the side ss.

The fractional parts may generally be estimated with an uncertainty of 0.1 to 0.2 by direct inspection; but more accurate measurements require suitable micrometric observation. The essential requirement for such micrometric observation may be met by an optical system forming a real image of the fringes and reference lines in a focal plane over which a filar micrometer observed by a low power ocular can be moved. Such apparatus can be readily improvised in any optical laboratory; but by far the best and most convenient method of measuring these fractions and observing the whole number of fringes is by means of the Pulfrich interferometer, exactly as constructed for use with the Fizeau method. ${ }^{13}$ This instrument also provides means for monochromatic illumination. It was with this instrument that all measurements at the Bureau of Standards have been made.

If a value of $L_{x}$ is required as in the computation of the expansion coefficient, it may be obtained by a mechanical contact micrometer. $L_{\mathrm{s}}$ may be obtained from $L_{\mathbf{x}}$ by equation (4).

\section{SENSITIVENESS, ACCURACY, AND RANGE}

\section{SENSITIVENESS}

By means of the Pulfrich instrument, $\delta n_{2}-\delta n_{1}$ may be determined as the mean of a small number of observations (say five) with an uncertainty, due to observational error, not greater than 0.01 or 0.02 . The instrument constant $K$ has the dimension of length. In the dilatometer which has actually been made $K=0.6$ micron. $\quad(D=28.7 \mathrm{I} \mathrm{mm} . d=\mathrm{x} 4.00 \mathrm{~mm} . \lambda=0.5876$ micron. $)$ The sensitiveness is, therefore, about 0.006 to 0.012 micron. It is possible to design an instrument of the same general type with a slightly but not much greater sensitiveness.

\section{ACCURACY}

Besides the error of observation above noted, the accuracy is limited only by unavoidable variation in contacts during the course of measurements. The consistency of Mr. Peters' extensive 


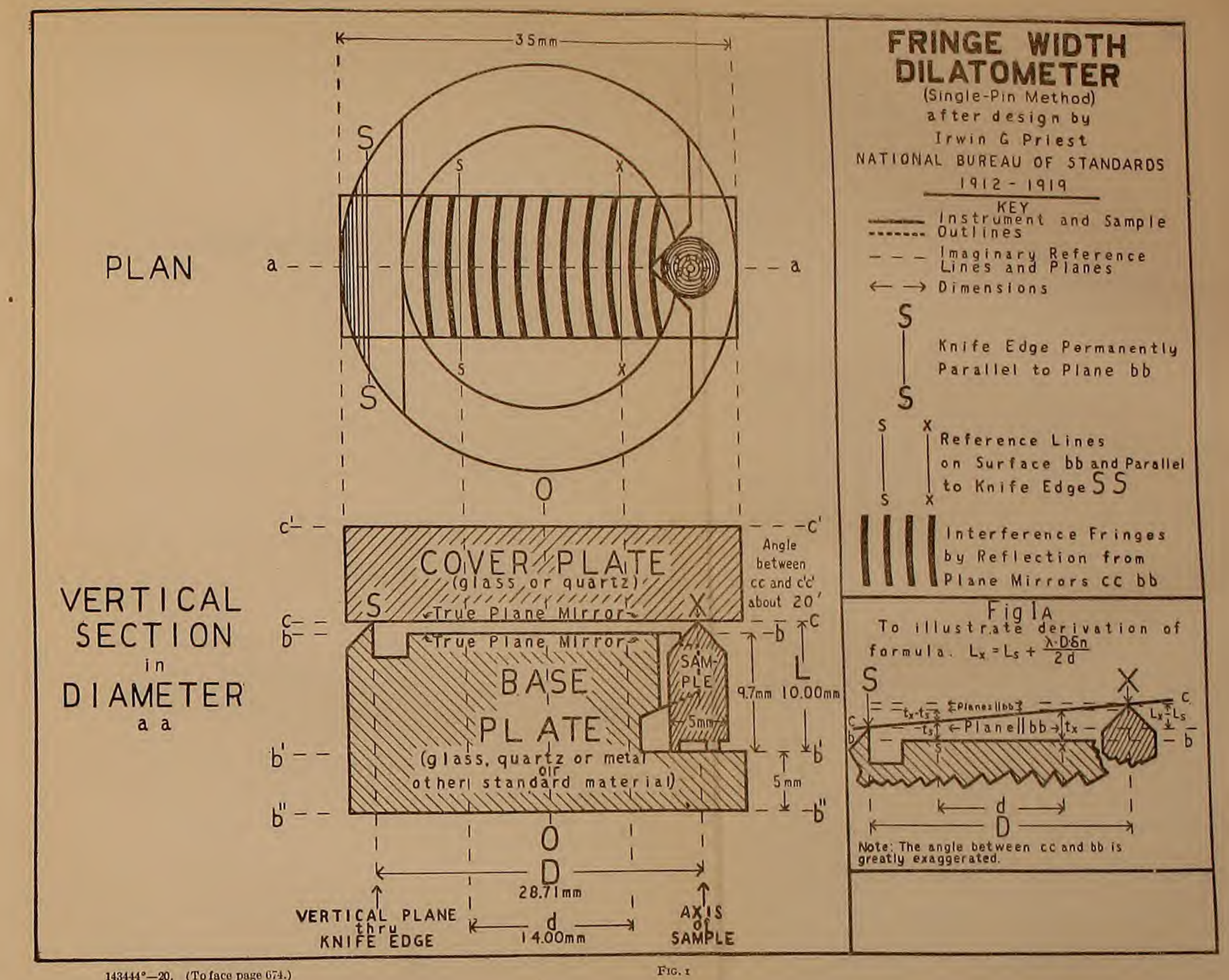

143444 $-20 . \quad$ (To face page 674.) 
measurements of thermal expansion of crystal quartz parallel and perpendicular to the axis, by this method and the Fizeau method, over an interval from about $26^{\circ} \mathrm{C}$ to $55^{\circ} \mathrm{C}$, shows that the determinations of $\Delta L_{\mathrm{x}}$ are actually reliable to about 0.01 or 0.02 micron. In these particular circumstances, therefore, the accuracy is nearly as good as the sensitiveness. Within the limits of its applicability, this method is found to be at least as accurate as the Fizeau method, and is probably more reliable. The contact errors must be separately considered under the particular circumstances of each investigation.

The determination of $K$ with sufficient accuracy to obtain in the computed result the full value of the accuracy attained in the measurement of $\delta n_{2}-\delta n_{1}$ presents no difficulty. For small values of $\delta n_{2}-\delta n_{1}$, it is evident that only a roughly approximate value of $K$ is required. In practical work as contemplated, $\delta n_{2}-\delta n_{1}$ will never be greater than 40 and will be determined with an uncertainty of about 0.02 or one part in 2000 . With the traveling microscope, $D$ and $d$ may be determined with about the same relative accuracy by careful observation. $\lambda$ is, of course, already known with far greater accuracy than required.

$L_{\mathrm{x}}$ may be obtained by a mechanical contact micrometer with the same relative accuracy with which $\Delta L_{x}$ is determined by the interferential measurements. $L_{\mathrm{s}}$ may usually be taken as equal to $L_{x}$ with sufficient accuracy.

\section{RANGE}

With the apparatus here described, the largest value of $\Delta L_{\mathrm{x}}-$ $\Delta L_{\mathrm{s}}$ which can well be measured is about 20 microns, in which case $\delta n_{1}$ and $\delta n_{2}$ are each about 20 and of opposite sign. It would be possible to increase the range slightly, but not very much.

\section{APPLICATIONS AND ADVANTAGES}

The application of this dilatometer to the determination of relative thermal expansivity, particularly in case the differential expansion is small, is obvious, and, as has been mentioned, it is already being used for this purpose. The base plate may be made of any material of standard expansivity and the various samples determined relative to it.

It appears that the instrument might also be adapted to the measurement of small increments in length, due to other causes, 
such as mechanical stress, magnetization, change in moisture content, etc.

The detailed methods will doubtless occur to those interested in such measurements; but it is well to point out in advance one important feature which might be overlooked. The absolute length of the sample is quite undetermined so far as the principle of the method is concerned. It is only necessary to construct the base plate with suitable distance between the planes $b b$ and $b^{\prime} b^{\prime}$ to accommodate the sample, the length of which may be fixed by other considerations. Thus, in magnetic tests it may be desirable to make this several centimeters instead of one.

The advantages of this method over the Fizeau method, ${ }^{14}$ which has been so widely used in measurements of thermal expansivity, may be enumerated as follows:

I. Smallness and simplicity of sample. Only one small pin is required, whereas the Fizeau method requires three similar pins, a ring 3 to $4 \mathrm{~cm}$ in diameter with three bearing points for the base plate and cover plate, or a block with one true plane surface about $10 \mathrm{~mm}$ in diameter. This advantage is important on three separate considerations, viz, ( $a$ ) economy of time and labor in preparing the sample; $(b)$ economy of material, which is important in some cases; $(c)$ the possibility of more minute study of homogeneity of material.

2. The correction for change of refractive index due to change in density of the air during the experiment is eliminated without evacuating the container. In the Fizeau method for thermal expansion, this is a very clumsy and troublesome although a small correction. ${ }^{15}$

3. The trouble of counting the passage of fringes, or obtaining their displacement by other tedious methods (observations on different wave lengths and solution of fraction puzzles) ${ }^{16}$ is entirely eliminated.

4. The chance of errors due to variation in contacts is reduced.

5. The possibility of displacement of the reference lines due to "creeping" or accidental displacement of the cover plate in the Fizeau apparatus is eliminated.

14 Fizeau, Ann. de Chim. et de Phy. (4), 2, p. $x_{4} 6$; 1864 .

${ }^{13}$ Pulfrich, Zeit. für Instk., 13, p. 456. Priest, Bulletin of the Bureaus of Standards, 9, D. 479.

16 Pulfrich, Zeit. für Instk., 13, pp. 369,437 , et seq. 


\section{CONSIDERATIONS OF MECHANICAL CONSTRUCTION: A TYPE OF SIMPLER CONSTRUCTION}

The theory of the construction of the base plate shown in Fig. $r$ is as follows:

I. A plate is made with plane and parallel surfaces, tested by Haidinger rings. ${ }^{17}$

2. All of one of these surfaces except a minute strip or edge forming the knife-edge $S$ is then ground away as shown in the figure. The knife-edge $S$ is thus, by construction, an element of the original surface, and so is parallel to the base $b^{\prime \prime} b^{\prime \prime}$, the other original surface.

3. The surface $b b$ is then ground and polished plane and parallel to $b^{\prime \prime} b^{\prime \prime}$ (by interference fringe tests) and about 0.2 to $0.3 \mathrm{~mm}$ below the original surface; and is thus, by construction, parallel to the knife-edge $S S$.

4. The recess to receive the sample is then ground into one side of the plate as shown leaving the surface $b^{\prime} b^{\prime}$ plane and parallel to $b^{\prime \prime} b^{\prime \prime}$ with good mechanical but not optical accuracy. (The disturbing reflection from $b^{\prime \prime} b^{\prime \prime}$ which results from this construction may be eliminated by cementing a plate of black glass onto $b^{\prime \prime} b^{\prime \prime}$.)

The construction was begun on this theory, but in the end it was found necessary, in order to save the piece, to slightly adjust the knife-edge. Indeed the middle portion of this edge has been cut away leaving only bearings near the ends to support the cover plate. There is no objection to this alteration.

This design is admittedly difficult to construct. The difficulty lies in the fact that the projecting knife-edge $S$ interferes greatly with the normal polishing process of correcting the surface $b b$ to an accurate true plane which is necessary. Although the piece which has actually been made is a credit to the optician, considering the difficulty of its construction, still the surface $b b$ is not as perfectly true plane as could be desired.

This difficulty can, of course, be obviated by making the expansion standard (a block with its base in the plane $b^{\prime} b^{\prime}$ and its apex at $S$, Fig. I) separate from the base plate. Indeed, several pieces of apparatus of this type have been made and used at the Bureau of Standards, the expansion standard consisting of two similar but separate posts or pins having their position deter-

${ }^{17}$ Müller-Pouillet, Lehrbuch der Physik, xoth ed., 2, book 3 (Optik), articles 35x-353. 
mined by contact with the base plate and lower mirror, just as the position of the sample is determined. In some cases the plate between $b^{\prime} b^{\prime}$ and $b^{\prime \prime} b^{\prime \prime}$ has been made separate from the lower mirror plate (the part between $b b$ and $b^{\prime} b^{\prime}$ ). The standard posts need not even be of the same material as the mirror plate.

The only disadvantage of this design is that the chance of contact errors and accidental lateral displacement of the points of support with reference to the reference lines on the lower mirror is introduced. These posts could, however, be mechanically bound in place, but this procedure has not actually been tried. Light spring clips might suffice. In the case of glass or fusedquartz apparatus, the fusion method of Parker and Dalladay ${ }^{18}$ might be used to secure the standard posts to the base plate.

The advisability of constructing special apparatus such as described in this paper will, of course, be determined by the extent of systematic work contemplated in any case. For a few experiments, it would not be worth while; but if large numbers of samples are to be examined, the special apparatus will soon "pay for itself" in convenience and economy of time. One of these standard instruments being ready, the preliminary adjustments for each separate determination will be simply:

I. Adjustment of diameter of sample to gage (mechanical caliper test) in order to give $D$ its standard value.

2. Adjustment of length of sample, $L_{x}$, to give fringes of suitable width. (The parallelism of the fringes to the reference lines $x x$ and $s s$ is fixed by construction of the instrument.)

Extensive data on the use of this apparatus have been obtained by C. G. Peters at this Bureau (See Sec. V, 2, above) and it is expected that he will publish these in the near future. In the meantime, the author would express his appreciation of Mr. Peters' work in developing and perfecting the method.

WASHINGTON, June 2 I, I9I9. 